\title{
Lymphatic Spread of Ovarian Cancer: Can the Anatomical and Pathological Knowledge Help a Personalized Treatment?
}

\author{
Giovanni D. Aletti, MD \\ Division of Gynecology, Department of Gynecologic Oncology, European Institute of Oncology, University of Milan, \\ Milan, Italy
}

Epithelial ovarian cancer has been recently classified into several different entities, according to the different pathogenesis. The traditional grading system of different histologic subtypes, which led to the concept of having different grade of the same tumor, has been changed over the past decades. A new system identifies different types with different prognosis and clinical behavior. ${ }^{1}$ Six main histologic subtypes were identified:

1. High-grade serous carcinoma

2. Low-grade serous carcinoma

3. Endometrioid carcinoma (which maintains a grading system)

4. Mucinous carcinoma (which maintains a grading system)

5. Clear cell carcinoma

6. Mixed/undifferentiated carcinoma

The primary routes of epithelial ovarian cancer dissemination are:

1. Through direct spread to adjacent organs, implantation of exfoliated cells on distant intraabdominal sites

2. Through the retroperitoneal lymphatic channels

The most recent version of the FIGO staging system recognizes the different impact on prognosis of lymph node metastases only (reclassified as stage IIIA1) as opposed to peritoneal macroscopic dissemination (stage IIIB-IIIC). ${ }^{2}$ In the previous classification, patients with nodal metastases

(C) Society of Surgical Oncology 2018

First Received: 24 March 2018;

Published Online: 25 April 2018

G. D. Aletti, MD

e-mail: giovanni.aletti@ieo.it

URL: http://www.ieo.it/ only were staged as stage IIIC and the same for grossly peritoneal involvement. While the rate of peritoneal involvement is more reflected by the final stage, the nodal involvement rate could be underestimated because of "inappropriate/incomplete" surgical staging for grossly early disease or inclusion of patients with nodal disease in the stage IIIC group.

The study reported in this issue from a well-established Gynecologic Group in Essen has two main merits ${ }^{3}$ :

1. The Essen Group has a well-established surgical quality program and well-established guidelines, which allowed, for the present study, a clear definition of the lymphatic spread according to different tumor stages and histologic subtypes

2. The utilization of the new histologic classification, which clearly identifies different entities, may suggest different prognostic and therapeutic implications

The main conclusions of the authors are:

1. Lymph nodal metastases are dependent on stage and histologic subtype, with high-grade serous harboring the higher rate and early-stage mucinous and lowgrade endometrioid having a negligible risk of nodal involvement.

2. Prognosis was worse in patients with lymph nodal metastases as opposed to patients without lymph node metastases, independent of histological subtype.

3. Given this results, the authors suggested that in patients with an incidental finding of an early mucinous or low-grade endometrioid adenocarcinoma, a restaging surgery might be discussed.

The main limitation of the study, as outlined by the authors, resides in the absence of data regarding the macroscopic involvement of the lymph nodes. Translated in clinical practice, removing grossly involved lymph 
nodes is part of a "debulking procedure," whereas removing clinically negative nodes is part of a "staging procedure."

The question remains about how to use the results outlined by the authors in clinical practice. In patients with advanced ovarian cancer, lymph nodes metastases were reported in $85.7 \%$ of all patients. Although the pathological status of the lymph nodes can give a prognostic information, this probably will not change the surgical approach or the need of lymphadenectomy in patients with advanced disease. In patients with suboptimal resection and bulky residual disease, performing a lymphadenectomy does not find a rational, considering that, for these patients, the main driver of survival is the peritoneal disease. Debulking of grossly involved nodes appears to be rational when a complete/nearly complete cytoreduction to no gross residual disease is obtained in the abdominal cavity, independent of histologic subtypes. The value of a complete pelvic and aortic lymphadenectomy in patients with advanced-stage disease and clinically negative nodes after a complete debulking has been recently defined. The results from the LION Trial (Lymphadenectomy in ovarian neoplasms-A prospective randomized AGO study group led gynecologic cancer intergroup trial) comparing systematic lymphadenectomy versus no lymphadenectomy in patients with advanced ovarian cancer debulked to no gross residual disease have been presented at ASCO Meeting in 2017. ${ }^{4}$ The median overall survival (69 and 66 months) and progression-free survival (26 months in both arms) were similar in the no-lymphadenectomy and lymphadenectomy groups. Given a higher perioperative morbidity and mortality in the lymphadenectomy arm, this procedure is no longer recommended in this setting of patients.

In early-stages disease, accurate retroperitoneal staging is recommended to define the correct extent of the disease at time of diagnosis. The main value of the procedure relies in the ability to potentially identify nodal metastases. In this way, an appropriate adjuvant treatment can be recommended according to the pathological findings. Inappropriate surgical staging can lead to understaging and subsequently inadequate postoperative treatment, which might ultimately worsen patients' prognosis.

A randomized trial comparing systematic pelvic and para-aortic lymphadenectomy to nodal sampling showed that the ability to detect nodal metastases was higher in the lymphadenectomy arm (22\% vs. 9\%). Less adjuvant treatment was given in the lymphadenectomy arm, and a trend towards a better progression-free (PFS) and overall survival (OS) was noted. ${ }^{5}$ A recent review examining the incidence of nodal metastases in 14 different studies showed that the mean incidence of pelvic/para-aortic nodal involvement in apparently stages I-II epithelial ovarian cancer is $14.2 \%$ (range $6.1-29.6 \%$ ). In grade 1 tumors, and in mucinous histology, the rate of nodal involvement is very low, almost negligible. ${ }^{6,7}$

In the present paper, the authors reported different rates of lymph nodal involvement in early-stage disease, according to different histologic subtypes. Confirming the previous findings, patients with low-grade endometrioid tumors and patients with mucinous tumors confined to the pelvis did not have any lymph node involvement. For this specific reason, several authors do not recommend to perform a systematic pelvic and para-aortic nodal dissection in these circumstances. The question remains whether to perform a lymphadenectomy on patients with different histologic subtypes and grading and clinically negative nodes. Many authors indicate that, if any adjuvant chemotherapy has to be performed (as in high grade serous carcinomas), a systematic lymphadenectomy is not necessary. According to these authors, the additional benefit of Paclitaxel to the Carboplatin regimen or the additional benefit of three more cycles of Carboplatin and Paclitaxel for microscopic nodal disease (upstaged patients) must be demonstrated. On the counterpart, if patients are truly stage I after a thorough peritoneal and retroperitoneal staging, this may result in less adjuvant treatment if not indicated. This might be true especially in less chemosensitive tumors, such as clear cell carcinomas or low-grade serous carcinomas. If grossly involved nodes are found in the preoperative workup or during the operation, these should be removed as part of a debulking procedure. This study certainly advances the knowledge regarding the retroperitoneal spreading according to different histologic subtypes. These results should be translated in a large collaborative effort to identify different subgroups of patients according to the different histologic subtypes and grading, who might need different staging procedures, and postoperative adjuvant treatments to generate a more personalized therapeutic approach for patients with early epithelial ovarian cancer.

\section{REFERENCES}

1. Shih Ie M, Kurman RJ. Ovarian tumorigenesis: a proposed model based on morphological and molecular genetic analysis. Am J Pathol. 2004;164(5):1511-8.

2. Prat J, FIGO Committee on Gynecologic Oncology. Staging classification for cancer of the ovary, fallopian tube, and peritoneum. Int J Gynaecol Obstet. 2014;124(1):1-5.

3. Heitz F, Harter P, Ataseven B, Heikaus S, Schneider S, et al. Stage and histologic subtype dependent frequency of lymph node metastases in patients with epithelial ovarian cancer undergoing systematic pelvic and para-aortic lymphadenectomy. Ann Surg Oncol. 2018; https://doi.org/10.1245/s10434-018-6412-y.

4. Harter P, Sehouli J, Lorusso D, Reuss A, Vergote I, et al. LION: Lymphadenectomy in ovarian neoplasms-a prospective randomized AGO study group led gynecologic cancer intergroup trial. Resulted reported at ASCO Meeting, Chicago, IL; 2017. 
5. Maggioni A, Benedetti Panici P, Dell'Anna T, et al. Randomised study of systematic lymphadenectomy in patients with epithelial ovarian cancer macroscopically confined to the pelvis. $\mathrm{Br} J$ Cancer. 2006;18:95(6):699-704.

6. Kleppe M, Wang T, Van Gorp T, Slangen BF, Kruse AJ, Kruitwagen RF. Lymph node metastasis in stages I and II ovarian cancer: a review. Gynecol Oncol. 2011;123(3):610-4.
7. Minig L, Heitz F, Cibula D, Bakkum-Gamez JN, Germanova A, Dowdy SC, et al. Patterns of lymph node metastases in apparent stage i low-grade epithelial ovarian cancer: a multicenter study. Ann Surg Oncol. 2017; 24(9):2720-6. 